\title{
The oncogenic effect of hepatitis C lasts beyond the disease's eradication
}

\author{
Sofia Cerqueira ${ }^{1}$, Catarina Teixeira ${ }^{2}$, Nuno Afonso ${ }^{2,3}$, Rui Castro ${ }^{1}$, Teresa Morgado ${ }^{1}$, Rui Alves ${ }^{2,3}$ \\ ${ }^{1}$ Nephrology Department, Centro Hospitalar de Trás-os-Montes e Alto Douro \\ ${ }^{2}$ Nephrology Department, Centro Hospitalar e Universitário de Coimbra \\ ${ }^{3}$ Nephrology, Faculty of Medicine, Coimbra, Portugal
}

\section{ABSTRACT}

Hepatitis C virus (HCV) is widely prevalent worldwide, with an estimated 180 million people infected. Its manifestations are not limited to hepatic disease, as it has widely known immunomodulator and oncogenic effects. Most HCV associated autoimmune and lymphoproliferative diseases have been shown to subside after successful antiviral treatment. However, viral eradication doesn't always equal lymphoma cure. In some cases, the oncogenic effect lasts well beyond viral elimination.

Although the new direct anti-viral agents (DAA) have proved to be safe and effective in treating HCV (with sustained viral responses of 91-95\%), this treatment has inherent toxicities. Further, immunologic manifestations of HCV-associated diseases, when present, may require broad immunosuppression in association with the antiviral treatment. We present a case that illustrates the complexities of treating these viral mediated immunologic and lymphoproliferative diseases.

Keywords: cryoglobulinemia; hepatitis C virus; lymphoma; onconephrology

\section{BACKGROUND}

Hepatitis $C$ virus can induce $B$ cell lymphocyte clonal proliferation that, in some cases, can lead to the development of a full-blown lymphoma. It has been suggested that in these cases viral eradication would lead to lymphoma cure. Nonetheless, some clones can maintain proliferation despite elimination of the viral stimuli. Treatment of these patients is extremely difficult and hazardous.

\section{CASE REPORT}

A 56-year-old male with no relevant clinical history, was admitted in March 2016 for a right-sided pleural effusion. Subsequent clinical investigation revealed a lymphoproliferative disease. Myelogram revealed $0.5 \%$ clonal $B$ cells for kappa chains, with non-Hodgkin lymphoma phenotype. Hepatitis C virus infection, genotype $3 a$, METAVIR fibrosis score $\mathrm{F} 4$, was also detected. The patient was observed at a hematology consultation and a watchful-waiting position was assumed.

Eight months after that, he was admitted due to acute renal lesion. There was no context of nephrotoxic medication. At that time, his serum creatinine was $2.1 \mathrm{mg} / \mathrm{dL}$; urine analysis was positive for hematuria and proteinuria (quantified at $2.9 \mathrm{~g} / 24 \mathrm{~h}$ ). Additional studies revealed diminished $\mathrm{C} 3$ and $\mathrm{C} 4$ and positive cryoglobulins with polyclonal IgG and monoclonal IgM; serum immune fixation was negative for a monoclonal component. Renal biopsy showed a membranoproliferative glomerulonephritis with immune complex deposition that was interpreted in association with the HCV infection.

After discussion with the Hepatology department, the patient was started on sofosbuvir and ribavirin for 24 weeks; prednisolone $1 \mathrm{mg} /$ $\mathrm{kg}$ was associated for treatment of the glomerulonephritis. During this 24-week period he developed severe infections (ophthalmic CMV, herpes zoster, acute tracheobronchitis), and still maintained diminished complement factors.

HCV treatment was finished, with sustained viral response (SVR) and later cure. However, at that time the myelogram showed an increased number of clonal B cells staining for kappa chains, with a lymphoplasmacytic lymphoma phenotype; there was continued production of cryoglobulins with complement consumption and persistent renal involvement.

After consulting with a hematologist, rituximab treatment was performed (two 1gr infusions, two weeks apart). Severe infectious episodes followed and there was persistent cryoglobulin production with complement consumption. Eventually, his chronic kidney disease progressed to end-stage and he started hemodialysis, 14 months after the kidney biopsy was performed.

Four months later he presented with necrotizing vasculitis in his lower limbs. Serum immune fixation then revealed a monoclonal IgM kappa gammopathy, and cryoglobulins showed only a monoclonal component. A new line of treatment with cyclophosphamide and corticosteroids was attempted and is now ongoing. 


\section{DISCUSSION}

After the advent of direct anti-viral agents (DAAs), precluding the need for interferon-based treatment of HCV, the safety and efficacy of this treatment has greatly improved. Pariente A et al ${ }^{1}$ reviewed 1123 HCV patients (mainly genotype 1 ) treated with DAAs in several centers in France, and witnessed an SVR at 12-24 weeks of 91\%. Likewise, the VALENCE study ${ }^{2}$ reported an SVR rate of $85 \%$ for patients treated with sofosbuvir and ribavirin for 24 weeks, with an incidence of around $1 \%$ for grade 3 or 4 adverse events. Although this regimen has proved to be safe, ribavirin is associated with some toxicity, namely anemia, fatigue, insomnia and nausea. Hence, this treatment regimen has been challenged by newer agents, such as daclatasvir and ledipasvir, which have shown good efficacy and safety profiles in recent studies ${ }^{3-5}$.

Although nowadays there is a wider experience in treating these patients with DAAs, not much is known about the outcomes of the subset of HCV patients with cryoglobulinemic vasculitis. The treatment in these patients needs to target not only virus eradication but also immunological quiescence. While in cases of mild to moderate vasculitis associated with HCV, an optimal antiviral DAA treatment should be given alone, in patients with severe vasculitis, rituximab or cyclophosphamide, with or without plasmapheresis are required, simultaneously with antiviral treatment. Corticosteroids are also helpful in controlling associated inflammation. Since this approach induces wide immunosuppression, one should weigh the benefits of starting an aggressive immunosuppressive treatment with the risk of causing serious infections and possibly HCV reactivation. We should also be aware that patients with cirrhosis appear to have a decreased ability to clear immune complexes. Cases of refractory cryoglobulinemic vasculitis should prompt the search for an underlying B-cell lymphoma.

HCV by itself, either with cryoglobulinemia or without, is believed to have a link with oncogenic diseases. The mechanism is not yet fully understood $^{6}$. Some authors support the idea that HCV causes an indirect transformation by which sustained antigenic stimulation causes inherent genomic instability ${ }^{7}$. This would lead from oligoclonal to monoclonal expansion and sometimes to lymphoma, probably through secondary oncogenic events ${ }^{7,8}$. In the interferon era, viral infection resolution was considered a central part of this disease treatment ${ }^{9}$, as cryoglobulinemic vasculitis and low-grade B cell lymphomas showed regression after antiviral treatment. Nonetheless, several cases have been described in which patients with HCV and cryoglobulinemic vasculitis did not respond to infection resolution alone ${ }^{10,11}$. Landau et al $^{12}$ argue that $B$ cell clones may need HCV to proliferate, but once this proliferation begins, it becomes independent from the viral antigen stimuli, a position that is sustained by other authors as well 7,8 . According to Landau et al's findings, it is rheumatoid factor and not viral antigen stimuli that is more closely associated with B cell activation. With HCV infection, this clone of $B$ cells becomes capable of avoiding deletion and does not respond to antigenic stimulation. There is also some evidence that HCV-associated lymphomas have unique molecular features and lack bcl2 translocations, augmenting their resistance to standard treatment ${ }^{13}$.

Treatment of HCV associated cryoglobulinemic vasculitis is not straightforward, as one needs to address the immunological as well as the viral infection feature. Although initially the main driver for disease progression is viral antigenic stimulation, in a further stage, the main drivers become the oncogenic and immunomodulatory properties of $\mathrm{HCV}$, well beyond its eradication.

We believe our case fully illustrated the aspects discussed above, in that a status of HCV carrier put in motion immune and lymphoproliferative mechanisms that went far beyond the viral infection and were sustained after its eradication. In retrospect, probably only an initial comprehensive chemotherapy aimed at the lymphoproliferative disease would be able to stop the disease process and avoid the progression of the kidney disease. Our piecemeal approach, conditioned by our justified concern with infectious events, only resulted in progressive selection of resistant B cell clones (as demonstrated by the evolution of the cryoglobulins from mixed polyclonal/monoclonal to strictly monoclonal) and disease progression.

As knowledge of the pathophysiology of this disease is expanding, our understanding of the main targets while treating it is constantly being modeled and (re)constructed. More investigation is needed in this field so that we can provide the optimal care with the best safety profile for our patients.

\section{Acknowledgments}

We would like to thank Coimbra University Medicine Faculty and the Nephrology Department of Coimbra Hospital.

Disclosure of potential conflicts of interest: none declared

\section{References}

1. Pariente A, Arpurt JP, Rémy AJ, et al. Hepatitis C treatment with all-oral direct-acting antivirals: Effectiveness and tolerance in a multicenter, prospective, observational study from French general hospitals (APROVVIE, ANGH). Presse Med 2019;48(3 Pt 1):e101-e110.

2. Zeuzem S, Geoffrey MD, Salupere R, et al. Sofosbuvir and ribavirin in HCV genotypes 2 and 3. N Engl J Med. 2014;370(21):1993-2001.

3. Sulkowski MS, David FG, Rodriguez-TorresM, et al. Daclatasvir plus sofosbuvir for previously treated or untreated chronic HCV infection. N Engl J Med 2014; 370:211-221.

4. Nelson DR, Cooper JN, Lalezari J P, et al. All-oral 12-week treatment with daclatasvir plus sofosbuvir in patients with hepatitis C virus genotype 3 infection: ALLY-3 phase III study. Hepatology, 61: 1127-1135.

5. Gane EJ, Hyland RH, An D, et al. Efficacy of ledipasvir and sofosbuvir, with or without ribavirin, for 12 weeks in patients with HCV genotype 3 or 6 infection. Gastroenterology, 2015 Nov;149(6):1454-1461.e1.

6. Brito, IP, Associação entre Linfomas Não Hodgkin de células B e infeção pelo vírus da Hepatite $C$, dissertação do Mestrado Integrado em Medicina, Instituto de Ciências Biomédicas Abel Salazar - Universidade do Porto, 2014.

7. Bachy E, Besson C, Suarez F et al. Hepatitis C Virus Infection and Lymphoma. Mediterr J Hematol Infect Dis. 2010; 2(1): e2010004.

8. Romero-Gomez M and Garcia-Romero D. Hepatitis C: crioglobulinémia y linfoma no-Hodgkin, Rev Esp Enferm Dig. 2008;100(3):164-70.

9. Gisbert GP, Garcia-Buey L, Pajares JM. Systematic review: regression of lymphoprolipherative disorders after treatment for hepatitis C infection, Aliment Pharmacol Ther. 2005;21:635-62.

10. Sollima $S$ et al, Persistent mixed cryoglobulinaemia vasculitis despite hepatitis $C$ virus eradication after interferon-free antiviral therapy, Rheumatology. 2016 November;55(11):2084-5.

11. Cornella SL, Stine JG, Kelly V, Caldwell SH, Shah NL. Persistence of mixed cryoglobulinemia despite cure of hepatitis $\mathrm{C}$ with new oral antiviral therapy including direct-acting antiviral sofosbuvir: A case series, Postgrad Med. 2015 May;127(4):413-7.

12. Landau DA, Saadoun D, Halfon P, et al. Relapse of hepatitis C virus-associated mixed cryoglobulinemia vasculitis in patients with sustained viral response, Arthritis Rheum. 2008;58:604-11.

13. Visco C,Wang J, Tisi MC, et al. Hepatitis C virus positive diffuse Large B-cell lymphomas have distinct molecular features and lack BCL2 translocations. Br J Cancer. 2017;117(11):1685-8.

\section{Correspondence to:}

Sofia Cerqueira, MD

Nephrology Department, Centro Hospitalar de Trás-os-Montes e Alto Douro Avenida da Noruega, 5000 Vila Real

E-mail: ssacerqueira@gmail.com 